\title{
Group Structures and Rectifiability in Powers of Spaces
}

by

\section{G. J. RIDDERBOS}

\author{
Presented by Czestaw BESSAGA
}

Summary. We prove that if some power of a space $X$ is rectifiable, then $X^{\pi w(X)}$ is rectifiable. It follows that no power of the Sorgenfrey line is a topological group and this answers a question of Arhangel'skir. We also show that in Mal'tsev spaces of pointcountable type, character and $\pi$-character coincide.

1. Introduction. A space $X$ is said to be rectifiable or to have a rectifiable diagonal if there is a homeomorphism $\Psi: X^{2} \rightarrow X^{2}$ and an element $e \in X$ such that $\pi_{1} \circ \Psi=\pi_{1}$ and for all $x \in X, \Psi(x, x)=(x, e)$. Here $\pi_{1}: X^{2} \rightarrow X$ is the projection onto the first co-ordinate. We call the mapping $\Psi$ a rectification of $X$, and the element $e$ is called a right unit element. We denote by $\pi w(X)$ and $w(X)$ the $\pi$-weight and weight of $X$ respectively, and by $\pi \chi(X), \chi(X)$ and $d(X)$ the $\pi$-character, character and density.

Spaces with a rectifiable diagonal were introduced by Uspenskiı $[16,17]$. Every topological group is rectifiable by means of the mapping $\Psi(x, y)=$ $\left(x, x y^{-1}\right)$. However, not every rectifiable space admits a group structure: the 7-dimensional sphere $S^{7}$ is rectifiable but not a topological group (see [17, $\S 3])$. Gul'ko proved in [6] that some results on topological groups generalize to rectifiable spaces. For example, a rectifiable space is metrizable if and only if it is first countable. Furthermore, some cardinal functions coincide on rectifiable spaces as they do on topological groups (see [6, Theorem 3.3] for more details).

The following question is due to A. V. Arhangel'skiu [1] and it was brought to my attention by T. Eisworth at the problem session of the Spring Topology and Dynamics Conference 2007:

2000 Mathematics Subject Classification: 54A25, 54B10, 54H11.

Key words and phrases: topological group, rectifiable, Mal'tsev space, products. 
QUESTION 1.1. Is some power of the Sorgenfrey line a topological group?

The aim of this note is to answer this question negatively. In fact, we will show that no power of the Sorgenfrey line is rectifiable. We will prove that if some power of the space $X$ is either rectifiable or a topological group, then $X^{\pi w(X)}$ has the same property. Since the Sorgenfrey line $\mathbb{S}$ has countable $\pi$-weight but $\mathbb{S}^{\omega}$ is not rectifiable, it follows that no power of $\mathbb{S}$ is rectifiable (see Corollary 2.4 below).

Our results are similar to those in [13]. Recall that a space $X$ is homogeneous if for every $x, y \in X$ there is a homeomorphism $h$ of $X$ such that $h(x)=y$. A space $X$ is called power homogeneous if $X^{\mu}$ is homogeneous for some cardinal number $\mu$. A space of the form $X^{\mu}$ is called $\Delta$-homogeneous if for all points $x$ and $y$ on the diagonal of $X^{\mu}$ there is a homeomorphism $h$ of $X^{\mu}$ mapping $x$ onto $y$. Here, the diagonal of $X^{\mu}$ consists of all points $x \in X^{\mu}$ such that $x_{\alpha}=x_{\beta}$ for all $\alpha, \beta \in \mu$. Ridderbos proved in [13, Theorem 4.5] that if $X$ is power homogeneous, then $X^{\pi w(X)}$ is $\Delta$-homogeneous. So the homogeneity of some (possibly very large) power of $X$ implies the existence of "many" homeomorphisms of $X^{\pi w(X)}$. Since every rectifiable space is homogeneous, the result of this paper may be seen as an improvement of Theorem 4.5 from [13] for the class of spaces $X$ that have a rectifiable power space. It is unknown in general whether $X^{\pi w(X)}$ is homogeneous whenever $X$ is power homogeneous (see [12, Question 2.4]).

We recall from [6] that not every homogeneous space is rectifiable. Gul'ko proved in [6] that no non-trivial space with the fixed point property is rectifiable. So the Hilbert cube is an example of a homogeneous space which is not rectifiable. The convergent sequence serves as an example of a nonhomogeneous space some power of which is a topological group and hence rectifiable.

2. Main result. All spaces under consideration are Hausdorff. All product spaces in this paper carry the usual product topology. Fix a space $X$. If $\mu$ is an infinite cardinal number and $A \subseteq \mu$, then we denote by $\pi_{A}$ the projection of $X^{\mu}$ onto $X^{A}$. If $\alpha \in \mu$, then we write $\pi_{\alpha}$ instead of $\pi_{\{\alpha\}}$. Furthermore, if $x \in X^{\mu}$ then $x_{A}=\pi_{A}(x)$ and $x_{\alpha}=\pi_{\alpha}(x)$.

For $A, B \subseteq \mu$, we use $\pi_{A, B}$ to denote the projection of $X^{\mu} \times X^{\mu}$ onto $X^{A} \times X^{B}$, i.e. $\pi_{A, B}(x, y)=\left(x_{A}, y_{B}\right)$. In this case we also abbreviate $\pi_{A, B}(w)$ by $w_{A, B}$, where $w=(x, y) \in X^{\mu} \times X^{\mu}$.

The following theorem was proved in [13].

Theorem 2.1 ([13, Theorem 4.4]). Let $X$ be a topological space and suppose that $\pi w(X) \leq \kappa \leq \mu$. Suppose further that $h: X^{\mu} \rightarrow X^{\mu}$ is a homeomorphism. If $B \in[\mu]^{\leq \kappa}$ then there are an $A \in[\mu]^{\leq \kappa}$ and a homeomorphism $h_{A}: X^{A} \rightarrow X^{A}$ such that $B \subseteq A$ and $h_{A} \circ \pi_{A}=\pi_{A} \circ h$. 
Now suppose that $X^{\mu}$ is rectifiable with rectification $\Psi$ and $\pi w(X)=$ $\kappa \leq \mu$. Then it follows from the above theorem that there are $A, B \in[\mu]^{\kappa}$ and a homeomorphism $\Psi_{A, B}: X^{A} \times X^{B} \rightarrow X^{A} \times X^{B}$ such that $\Psi_{A, B} \circ \pi_{A, B}=$ $\pi_{A, B} \circ \Psi$. To prove our main result, we need $A=B$. We prove that this is possible in the following lemma.

Lemma 2.2. Let $X$ be a topological space and suppose that $\pi w(X) \leq \kappa$ $\leq \mu$. Suppose further that $h: X^{\mu} \times X^{\mu} \rightarrow X^{\mu} \times X^{\mu}$ is a homeomorphism. Then there are an $A \in[\mu]^{\kappa}$ and a homeomorphism $h_{A}: X^{A} \times X^{A} \rightarrow X^{A} \times X^{A}$ such that $h_{A} \circ \pi_{A, A}=\pi_{A, A} \circ h$.

Proof. The proof is almost identical to the proof of Theorem 4.4 in [13]. Applying [13, Theorem 4.3], we find sequences $\left(A_{n}\right)_{n}$ and $\left(B_{n}\right)_{n}$ such that

(1) $A_{0}=B_{0} \in[\mu]^{\kappa}$ are arbitrary and $A_{n}, B_{n} \in[\mu]^{\kappa}$ for all $n<\omega$,

(2) $A_{n} \cup B_{n} \subseteq A_{n+1} \cap B_{n+1}$,

(3) for all $w, z \in X^{\mu} \times X^{\mu}$,

$$
w_{A_{n}, B_{n}}=z_{A_{n}, B_{n}} \Leftrightarrow h(w)_{A_{n}, B_{n}}=h(z)_{A_{n}, B_{n}} .
$$

Finally, we let $A=\bigcup_{n<\omega} A_{n}$ and $B=\bigcup_{n<\omega} B_{n}$. It follows from (2) that $A=B$ and by (3) we have, for all $w, z \in X^{\mu} \times X^{\mu}$,

$$
w_{A, A}=z_{A, A} \Leftrightarrow h(w)_{A, A}=h(z)_{A, A} .
$$

We now take any injection $i: X^{A} \rightarrow X^{\mu}$ such that $\pi_{A} \circ i=\mathrm{id}$ and we let $h_{A}=\pi_{A, A} \circ h \circ(i \times i)$. The proof that $h_{A}$ is a homeomorphism with the desired properties is exactly as in the proof of [13, Theorem 4.4].

We now come to our main result.

THEOREM 2.3. Suppose that some power of $X$ is either rectifiable or a topological group. Then $X^{\pi w(X)}$ has the same property.

Proof. Let $\pi w(X)=\kappa$ and suppose that $X^{\mu}$ is either rectifiable or a topological group. It is easy to see that the product of rectifiable spaces is again rectifiable, and the same is true for topological groups (cf. [7, Theorem 6.2]). We may therefore assume without loss of generality that $\kappa \leq \mu$.

We first deal with the rectifiable case. So assume that $\Psi$ is a rectification of $X^{\mu}$ with right unit element $e \in X^{\mu}$. By Lemma 2.2 we find $A \in[\mu]^{\kappa}$ and a homeomorphism $\Psi_{A}$ on $X^{A} \times X^{A}$ such that

$$
\Psi_{A} \circ \pi_{A, A}=\pi_{A, A} \circ \Psi \text {. }
$$

It follows from $(\dagger)$ that $\Psi_{A}$ is a rectification on $X^{A}$ with right unit element $e_{A}$. To see this, fix any injection $i: X^{A} \rightarrow X^{\mu}$ such that $\pi_{A} \circ i=\mathrm{id}$. Then if $x \in X^{A}$ is arbitrary, we have

$$
\begin{aligned}
\Psi_{A}(x, x) & =\Psi_{A} \circ \pi_{A, A}(i(x), i(x))=\pi_{A, A} \circ \Psi(i(x), i(x)) \\
& =\pi_{A, A}(i(x), e)=\left(x, e_{A}\right) .
\end{aligned}
$$


Next, let $\pi_{A}^{1}$ and $\pi_{\mu}^{1}$ be the projections of $X^{A} \times X^{A}$ and $X^{\mu} \times X^{\mu}$ onto the first co-ordinate $X^{A}$ and $X^{\mu}$ respectively. Since $\pi_{\mu}^{1} \circ \Psi=\pi_{\mu}^{1}$, we also have $\pi_{A}^{1} \circ \pi_{A, A} \circ \Psi=\pi_{A}^{1} \circ \pi_{A, A}$. Hence

$$
\begin{aligned}
\pi_{A}^{1} \circ \Psi_{A} & =\pi_{A}^{1} \circ \Psi_{A} \circ \pi_{A, A} \circ(i \times i)=\pi_{A}^{1} \circ \pi_{A, A} \circ \Psi \circ(i \times i) \\
& =\pi_{A}^{1} \circ \pi_{A, A} \circ(i \times i)=\pi_{A}^{1} .
\end{aligned}
$$

This shows that $\Psi_{A}$ is a rectification on $X^{A}$ with unit element $e_{A}$. Since $|A|=\pi w(X)$, it follows that $X^{\pi w(X)}$ is rectifiable.

Next we deal with the case that $X^{\mu}$ is a topological group with identity $e \in X^{\mu}$. We denote the group operation on $X^{\mu}$ by $\star$ and define $g: X^{\mu} \times X^{\mu}$ $\rightarrow X^{\mu} \times X^{\mu}$ by $g(x, y)=\left(x^{-1}, x \star y\right)$. Then $g$ is a homeomorphism so we may apply Lemma 2.2 to find $A \in[\mu]^{\kappa}$ and a homeomorphism $g_{A}$ on $X^{A} \times X^{A}$ such that

$$
g_{A} \circ \pi_{A, A}=\pi_{A, A} \circ g .
$$

Next let $\pi_{A}^{1}$ and $\pi_{A}^{2}$ be the projections of $X^{A} \times X^{A}$ onto the respective co-ordinates. We now define a continuous binary operation $\star_{A}$ on $X^{A}$ as follows: for $x, y \in X^{A}$,

$$
x \star_{A} y:=\pi_{A}^{2} \circ g_{A}(x, y) .
$$

It is not hard to deduce from (

$$
\star_{A} \circ \pi_{A, A}=\pi_{A} \circ \star
$$

We leave it to the reader to verify that $\star_{A}$ is a continuous group operation on $X^{A}$ with identity $e_{A}$ and inverses given by $h$ where $h(x)=\pi_{A}^{1} \circ g_{A}\left(x, e_{A}\right)$. Since $|A|=\pi w(X)$, this completes the proof.

It follows from the corollary below that no power of the Sorgenfrey line is rectifiable; simply note that the $\pi$-weight of this space is countable whereas it has uncountable weight. This also answers Question 1.1.

Corollary 2.4. Suppose that some power of $X$ is rectifiable. Then:

(1) $w(X)=\pi w(X)$,

(2) if the $\pi$-weight of $X$ is countable, then $X$ is metrizable.

Proof. It follows from the previous theorem that $X^{\pi w(X)}$ is rectifiable. $\mathrm{Gul}^{\prime}$ ko proved in [6, Theorem 3.3] that the $\pi$-weight and weight coincide in rectifiable spaces. Since $w\left(X^{\pi w(X)}\right)=w(X)$ and $\pi w\left(X^{\pi w(X)}\right)=\pi w(X)(\mathrm{cf}$. $[8,5.3])$, this proves the first statement.

Next suppose that the $\pi$-weight of $X$ is countable. Then it follows from (1) and $[8,5.3]$ that the character of $X^{\omega}$ is countable. Since $X^{\omega}$ is rectifiable it follows from [6, Theorem 3.2] that $X^{\omega}$ (and hence $X$ ) is metrizable.

Van Mill noted in [9, Corollary 1.3] that if $X$ is a homogeneous compactum, then assuming $\mathrm{GCH}, \chi(X) \leq \pi w(X)$. It follows from a result of 
Arhangel'skiř, van Mill and Ridderbos that the same statement holds if $X$ is a power homogeneous compactum (see [3, Corollary 2.5] and [11, Proposition 2.1]). If some power of $X$ is rectifiable, then we can drop the compactness assumption and GCH entirely, since in this case it follows from Corollary 2.4(1) that $\chi(X) \leq \pi w(X)$. Since the $\pi$-character and character coincide in rectifiable spaces by [6, Theorem 3.3], this raises the following question:

QUESTION 2.5. Suppose that some power of $X$ is rectifiable (or a topological group $)$. Is it the case that $\pi \chi(X)=\chi(X)$ ?

We will prove below that for spaces of point-countable type, the answer to this question is "YES". Recall that a space $X$ is called a Mal'tsev space if there is a continuous function $\mu: X^{3} \rightarrow X$ such that $\mu(x, x, y)=y=\mu(y, x, x)$ for all $x, y \in X$. In this case the function $\mu$ is called a Mal'tsev function.

The class of Mal'tsev spaces is closed under taking retractions, and if $X$ is a topological group, then the formula $x y^{-1} z$ defines a Mal'tsev function on $X$. So if a space $X$ is a retract of a topological group, then it is a Mal'tsev space. Conversely, it was shown by Sipacheva in [15] that every compact $\mathrm{Mal}^{\prime}$ tsev space is also a retract of a topological group (see [5, Corollary 6] and [10, Theorem 1.6] for generalizations).

Uspenskiu noted in [17, Proposition 14] that rectifiable spaces admit a Mal'tsev operation. So if some power of a space $X$ is rectifiable, then $X$ is itself a Mal'tsev space. Now let $X$ be a compact space some power of which is rectifiable. Then $X$ is a compact $\mathrm{Mal}^{\prime}$ tsev space and therefore $\chi(X)=$ $\pi \chi(X)$. This follows from the fact that compact Mal'tsev spaces are dyadic, which was proved by Uspenskiı̌ [17], and therefore weight and $\pi$-character coincide in compact Mal'tsev spaces (see [2, III, §1]). We shall now give an elementary proof of the fact that character and $\pi$-character coincide on compact Mal'tsev spaces and in fact this is also true for Mal'tsev spaces of point-countable type. We first prove the following theorem.

TheORem 2.6. Suppose that $X$ is a compact subset of a Mal'tsev space $Z$. Then for all $x \in X, \chi(x, X) \leq \pi \chi(x, Z)$.

Proof. Fix a Mal'tsev function $\mu$ on $Z$. All closures are taken in $Z$. Fix a point $x \in X$ and a local $\pi$-base $\mathcal{B}$ at $x$ in $Z$. For every $B \in \mathcal{B}$, we pick $y_{B} \in B$. For $y \in Z$ we define $f_{y}: Z \rightarrow Z$ by $f_{y}(z)=\mu(y, x, z)$. Note that $f_{y}(x)=y$ for every $y \in Z$.

Let $\mathcal{U}=\left\{X \cap f_{y_{B}}^{-1}[B]: B \in \mathcal{B}\right\}$. We will show that this is a local basis at $x$ in $X$. Since $|\mathcal{U}| \leq|\mathcal{B}|$, this suffices to prove the theorem. Note in particular that $\mathcal{U}$ is a collection of open neighbourhoods of $x$ in $X$.

So let $U$ be some open neighbourhood of $x$ in $X$. Then $X \backslash U$ is a compact subset of $Z$ which misses $x$ and therefore we may find an open neighbourhood 
$V$ of $x$ in $Z$ such that

$$
X \backslash U \subseteq Z \backslash \bar{V}
$$

It follows that

$$
\{x\} \times\{x\} \times(X \backslash U) \subseteq \mu^{-1}[Z \backslash \bar{V}] .
$$

By a standard compactness argument, we may find an open neighbourhood $W$ of $x$ in $Z$ such that

$$
W \times W \times(X \backslash U) \subseteq \mu^{-1}[Z \backslash \bar{V}] .
$$

Since $\mathcal{B}$ is a local $\pi$-base at $x$ in $Z$, we may find $B \in \mathcal{B}$ such that $B \subseteq W \cap V$. We prove the following claim;

Claim 1. If $y \in B$, then $X \cap f_{y}^{-1}[B] \subseteq U$.

Proof of Claim. Let $z \in X \cap f_{y}^{-1}[B]$ and suppose that $z \notin U$. Then $z \in X \backslash U$ and since $x, y \in W$, it follows that $(y, x, z) \in W \times W \times(X \backslash U)$. This implies that $f_{y}(z)=\mu(y, x, z) \in Z \backslash \bar{V}$. So we have $f_{y}(z) \in B \cap(Z \backslash \bar{V})$. But $B \subseteq V$ and therefore $B \cap(X \backslash \bar{V})=\emptyset$. This is a contradiction.

From the claim it follows that $X \cap f_{y_{B}}^{-1}[B]$ is a neighbourhood of $x$ in $X$ which is contained in $U$. Since $U$ was arbitrary, we have proved that $\mathcal{U}$ is a local basis at $x$ in $X$, and this completes the proof.

Recall that the point compactness type of a space $X, \operatorname{pct}(X)$, is defined as the least cardinal number $\kappa$ with the property that for every $x \in X$, there is a compact subset $F$ of $X$ such that $x \in F$ and $\chi(F, X) \leq \kappa$. A space is said to be of point-countable type if its point compactness type is countable. It follows from the corollary below that if $X$ is of point-countable type and some power of $X$ is rectifiable, then $\chi(X)=\pi \chi(X)$.

Corollary 2.7. If $X$ is a Mal'tsev space, then $\chi(X)=\pi \chi(X) \operatorname{pct}(X)$. In particular, if $X$ is of point-countable type, then $\chi(X)=\pi \chi(X)$.

Proof. Fix $x \in X$ and a compact subset $F$ of $X$ such that $x \in F$ and $\chi(F, X) \leq \operatorname{pct}(X)$. We have just proved that $\chi(x, F) \leq \pi \chi(x, X)$. Furthermore, it is well known that $\chi(x, X) \leq \chi(x, F) \cdot \chi(F, X)$ (see for example [4, 3.1.E]), so it follows that

$$
\chi(x, X) \leq \chi(x, F) \cdot \chi(F, X) \leq \pi \chi(x, X) \operatorname{pct}(X) .
$$

This shows that $\chi(X) \leq \pi \chi(X) \operatorname{pct}(X)$. The reverse inequality is always valid, so this completes the proof.

The following corollary improves [6, Theorem 3.4].

Corollary 2.8. If $X$ is a Mal'tsev space of point-countable type, then $\chi(X)=t(X)$.

Proof. This follows from the previous corollary and the fact that $\pi \chi(X)$ $\leq t(X)$ for spaces $X$ of point-countable type (see $\left[14\right.$, Theorem $\left.1^{\prime}\right]$ ). 


\section{References}

[1] A. V. Arhangel'skiǔ, private communication.

[2] - , The structure and classification of topological spaces and cardinal invariants, Uspekhi Mat. Nauk 33 (1978), no. 6, 29-84, 272 (in Russian).

[3] A. V. Arhangel'skiř, J. van Mill, and G. J. Ridderbos, A new bound on the cardinality of power homogeneous compacta, Houston J. Math. 33 (2007), 781-793.

[4] R. Engelking, General Topology, 2nd ed., Sigma Ser. Pure Math. 6, Heldermann, Berlin, 1989.

[5] P. M. Gartside, E. A. Reznichenko, and O. V. Sipacheva, Mal'tsev and retral spaces, Topology Appl. 80 (1997), 115-129.

[6] A. S. Gul'ko, Rectifiable spaces, ibid. 68 (1996), 107-112.

[7] E. Hewitt and K. A. Ross, Abstract Harmonic Analysis. Vol. I: Structure of Topological Groups, Integration Theory, Group Representations, Grundlehren Math. Wiss. 115, Academic Press, New York, 1963.

[8] I. Juhász, Cardinal Functions in Topology-Ten Years Later, 2nd ed., Math. Centre Tracts 123, Math. Centrum, Amsterdam, 1980.

[9] J. van Mill, On the character and $\pi$-weight of homogeneous compacta, Israel J. Math. 133 (2003), 321-338.

[10] E. A. Reznichenko and V. V. Uspenskir, Pseudocompact Mal'tsev spaces, Topology Appl. 86 (1998), 83-104.

[11] G. J. Ridderbos, Cardinality restrictions on power homogeneous $T_{5}$ compacta, Studia Sci. Math. Hungar., to appear.

[12] -, A characterization of power homogeneity, Topology Appl., to appear.

[13] -, On the cardinality of power homogeneous Hausdorff spaces, Fund. Math. 192 (2006), 255-266.

[14] B. Shapirovskiı̌, $\pi$-character and $\pi$-weight in bicompacta, Dokl. Akad. Nauk SSSR 223 (1975), 799-802 (in Russian).

[15] O. V. Sipacheva, Compacta with a continuous Mal'tsev operation and retracts of topological groups, Vestnik Moskov. Univ. Ser. I Mat. Mekh. 1991, no. 1, 33-36, 104 (in Russian).

[16] V. V. Uspenskiur, The Mal'tsev operation on countably compact spaces, Comment. Math. Univ. Carolin. 30 (1989), 395-402.

[17] —, Topological groups and Dugundji compacta, Mat. Sb. 180 (1989), 1092-1118 (in Russian); English transl.: Math. USSR-Sb. 67 (1990), 555-580.

\section{G. J. Ridderbos}

Faculty of Sciences

Division of Mathematics

Vrije Universiteit

De Boelelaan $1081^{\mathrm{A}}$

1081 HV Amsterdam, the Netherlands

E-mail: gfridder@few.vu.nl

http://www.math.vu.nl/ gfridder

Received May 22, 2007;

received in final form September 27, 2007 